\title{
The Role of Calponin in Cell Mechano transduction
}

\author{
Zhiping Miao and Airong Qian* \\ School of Life Sciences, Northwestern Polytechnic University, Xi'an, China
}

Received: February 04, 2018; Published: February 15, 2018

*Corresponding author: Airong Qian, School of Life Sciences, Northwestern Polytechnic University, China, Tel: 86-29-88491840; Fax: +86-29-88491840, Email: qianair@nwpu.edu.cn

\begin{abstract}
H2-calponin, an actin-cytoskeleton associated protein, is expressed in smooth muscle and certain non-muscle cells. Researchers have demonstrated that $\mathrm{H} 2$-calponin proteins are highly expressed in developing and remodeling tissues. As a mechanical tension responsive gene inside the cell, H2-calponin interacts with F-actin and certain focal adhesion proteins, modifies cytoskeleton function, takes part in mechanochemical signal transmission and plays an important role in cell proliferation, differentiation, motility and cytokinesis. Based on recent research progresses in this field, this paper reviewed the molecular characteristics of $\mathrm{H} 2$-calponin and the role of $\mathrm{H} 2$-calponin in mechano sensation and mechano transduction.
\end{abstract}

Keywords: H2-calponin; Cytoskeleton; Mechano transduction; Mechanical stress

\section{Introduction}

Calponin is a member of the family of actin-binding proteins found in a variety of cells, which is well known for its inhibition of actin-activated myosin ATPase. There are three distinct calponin is forms in the vertebrates as the products of three homologous genes, named h1-calponin (basic calponin, iso electric point (pI) = 9.4), h2-calponin (neutral calponin, pI = 7.5), and h3-calponin (acidic calponin, $\mathrm{pI}=5.2$ ) [1-3]. The amino acid sequences of the three calponin is forms are highly conserved in most parts of the polypeptide chain [4], suggesting that in smooth muscle and non-muscle cells they may function by regulating the structure of actin filaments. In recently, high levels of h2-CaP in non-muscle cells have been found including keratinocytes [5,6], epidermal cells [7,8], endothelial cells [9], fibroblasts [2], macrophages and myeloid phagocytes [10]. H2-CaP may play an important role in the organization of actin cytoskeleton [11], proliferation [12], and migration [10] and in cytokinesis [12]. h2-CaP is expressed in lung alveolar epithelial cells, epidermal keratinocytes, and fibroblasts and plays a part in stabilizing the actin cytoskeleton. The expression and degradation of $\mathrm{h} 2-\mathrm{CaP}$ are regulated by mechanical tension in the cytoskeleton [7, 8]. Extended from calponin's role in proliferation, migration, development and in cytokinesis studies, this review focuses on the role of calponin in cell mechano transduction.

\section{H2-Calponin and Mechanical Tension}

In vitro calponin's function is regulated by its interaction with Ca2+- binding protein and/or by its phosphorylation. This suggests that calponin may play an important role in signal transduction from the membrane receptor to the contractile proteins in smooth muscle. Cells transmit mechanical signaling to inside mainly through microvilli or ion channel on the cell membrane. Danninger et al. [11] showed that h2 calponin localizes to the ends of stress fibres and in the motile lamellipodial protrusions of spreading cells. The localization of h2 calponin to the actin filament may facility cells to sense stress. This preferred association of $\mathrm{h} 2$ calponin with $\mathrm{F}$-actin at the cell periphery suggests that h2 CaP may be involved in the regulation of actin organization. Fukui et al. [5] demonstrated the presence of h2-calponin in human keratinocytes, and it might play a role in the structural organization of actin cytoskeleton at the cytoplasmic region of cell-to-cell junctions of keratinocytes. H2calponin may represent a novel manifestation of mechanical tension responsive gene regulation that may modify cytoskeleton function [8]. The expression of h2-calponin is cell anchorage-dependent and h2-calponin expression decreases when cells are rounded up and remain low when cells are prevented from adhering to culture dish. After the floating cells are allowed to form a monolayer in plastic dish, h2-calponin expression recovers. H2-calponin expression is affected by the mechanical properties of the culture matrix. When cells are cultured on soft polyacrylamide gel which applies less traction force to the cell and, therefore, lower mechanical tension in the cytoskeleton, the level of h2-calponin is significantly lower than that in cells cultured on hard gel. The results indicated that mechanical tension in the cytoskeleton produced against the substrate stiffness [13] regulates h2-calponin gene expression. Hossain et al demonstrated that the expression of h2-calponin is rapidly up-regulated during postnatal lung development coincident with the respiratory expansion of alveolar epithelial cells [7]. The mechanical tension built in the actin filaments by myosin motors 
regulates h2-calponin gene expression. Blebbistatin, an inhibitor of non-muscle myosin II, significantly decreased the levels of h2-calponin protein [8] and mRNA [7] in comparison with the controls by diminishing the mechanical tension built in the actin cytoskeleton [14].

\section{H2-Calponin and Mechanotransduction}

All living cells respond to mechanical forces by morphology alteration, gene regulation and protein modifications. The reorganization of the actin cytoskeleton plays a key role in cellular responses to mechanical stimuli. Therefore, the changes in localization and expression of $\mathrm{h} 2$-calponin in cells may represent a mechanism for the actin cytoskeleton to respond to the mechanical environment of the cell. However, how cells sense mechanical signals and convert them into biochemical regulations during cellular responses to mechanical stimuli are much less understood. The process of activating myosin ATPase to generate contractile force in muscle may be involved in the conversion of chemical signals into mechanical responses in living cells. Mechanical signals to bone are introduced to osteoblasts or osteocytes by mechanosensing receptors (such as integrins) [15]. H1 calponin is found in osteoblasts and as a negative regulator of new bone formation [16]. H1 calponin-deficient mice showed enhanced membranous bone formation in their developmental stage, which results in thick long bones at birth. In $\mathrm{h} 1$ calponin deficient mice, the degree of tailsuspension induced bone loss was significantly alleviated and the phosphorylation form of $\mathrm{h} 1$ calponin might be a causative molecule in weightless-induced osteopenia [16].

\section{Conclusion}

In conclusion, there are three iso forms of calponin in smooth muscle and non-muscle cells and the function of calponin as regulators of the actin cytoskeleton. Recent experimental data demonstrated calponin may represent a novel manifestation of mechanical tension or abnormal gravity responsive gene regulation that may modify cytoskeleton function to involve in cell mechanosensation and mechnotransduction. The detailed mechanism on how cells sense mechanical stimuli and transmit the mechanical signals to biochemical signals needs to be further studied in the future. Further investigations into function of calponin in cell mechanotransduction under physiological and pathological conditions will not only provide fundamental knowledge for cell mechanosensation, but also help to understand the development and treatment of diseases.

\section{References}

1. Strasser P, Gimona M, Moessler H, Herzog M, Small JV (1993) Mammalian calponin identification and expression of genetic variants. FEBS Letters 330(1): 13-18.

2. Hossain MM, Zhao G, Woo MS, Wang JH, Jin JP (2016) Deletion of Calponin 2 in Mouse Fibroblasts Increases Myosin II-Dependent Cell Traction Force. Biochemistry 55(43): 6046-6055.

3. Jin JP, Wu D, Gao J, Nigam R, Kwong S (2003) Expression and purification of the h1 and h2 isoforms of calponin. Protein Expression and Purification 31(2): 231-239.

4. Jin JP, Zhang ZL, Bautista JA (2008) Isoform diversity, regulation, and functional adaptation of troponin and calponin. Critical Review in Eukaryotic Gene Expression 18(2): 93-124.

5. Fukui Y, Masuda H, Takagi M, Takahashi K, Kiyokane K (1997) The presence of h2-calponin in human keratinocyte. J Dermatol Sci 14(1): 29-36.

6. Wu KC, Jin JP (2008) Calponin in non-muscle cells. Cell Biochem Biophys 52(3): 139-148.

7. Hossain MM, Smith PG, Wu K, Jin JP (2006) Cytoskeletal tension regulates both expression and degradation of h2-calponin in lung alveolar cells. Biochemistry 45(51): 15670-15683.

8. Hossain MM, Crish JF, Eckert RL, Lin JJ, Jin JP, et al. (2005) H2-Calponin is regulated by mechanical tension and modifies the function of actin cytoskeleton. J Biol Chem 280(51): 42442-42453.

9. Tang J, Hu G, Hanai J, Yadlapalli G, Lin Y (2006) A critical role for calponin 2 in vascular development. J Biol Chem 281(10): 6664-6672.

10. Huang QQ, Hossain MM, Sun W, Xing L, Pope RM, et al. (2016) Deletion of calponin 2 in macrophages attenuates the severity of inflammatory arthritis in mice. Am J Physiol Cell Physiol 311(4): C673-C685.

11. Danninger C, Gimona M (2000) Live dynamics of GFP-calponin: isoformspecific modulation of the actin cytoskeleton and autoregulation by C-terminal sequences. J Cell Sci 113: 3725-3736.

12. Hossain MM, Hwang DY, Huang QQ, Sasaki Y,Jin JP (2003) Developmentally regulated expression of calponin isoforms and the effect of h2-calponin on cell proliferation. Am J Physiol, 284(1): 156-167.

13. Discher DE, Janmey P, Wang YL (2005) Tissue cells feel and respond to the stiffness of their substrate. Science 310(5751): 1139-1143.

14. Griffin MA, Sen S, Sweeney HL, Discher DE (2004) Adhesion contractile balance in myocyte differentiation. Journal of Cell Science 117(Pt24): 5855-5863.

15. Gluhak Heinrich J, Ye L, Bonewald LF, Feng JQ MacDougall M, et al. (2003) Mechanical loading stimulates dentin matrix protein 1 (DMP1) expression in osteocytes in vivo. J Bone Miner Res 18(5): 807-817.

16. Yotsumoto N, Takeoka M, Yokoyama M (2010) Tail suspended mice lacking calponin $\mathrm{H} 1$ experience decreased bone loss. Tohoku J Exp Med 221(3): 221-227.

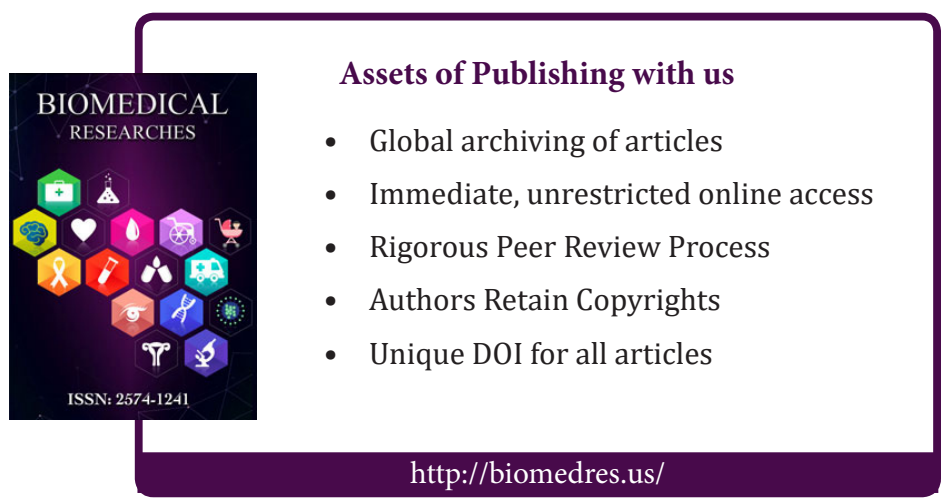

